\title{
A solar mean field dynamo benchmark
}

\author{
L. Jouve ${ }^{1}$, A. S. Brun ${ }^{1}$, R. Arlt ${ }^{2}$, A. Brandenburg ${ }^{3}$, M. Dikpati ${ }^{4}$, A. Bonanno ${ }^{5}$, P. J. Käpylä ${ }^{3,7}$, \\ D. Moss ${ }^{6}$, M. Rempel ${ }^{4}$, P. Gilman ${ }^{4}$, M. J. Korpi ${ }^{7}$, and A. G. Kosovichev ${ }^{8}$ \\ ${ }^{1}$ Laboratoire AIM, CEA/DSM-CNRS-Université Paris Diderot, DAPNIA/SAp, 91191 Gif sur Yvette, France \\ e-mail: ljouve@cea.fr \\ 2 Astrophysikalisches Institut Potsdam, An der Sternwarte 16, 14482 Potsdam, Germany \\ 3 Nordita, AlbaNova University Center, Roslagstullsbacken 23, 10691 Stockholm, Sweden \\ ${ }^{4}$ High Altitude Observatory, National Center for Atmospheric Research, 3080 Center Green, Boulder, CO 80301, USA \\ 5 INAF Osservatorio Astrofisico di Catania, via S.Sofia 78, 95123 Catania, Italy \\ 6 School of Mathematics, University of Manchester, Manchester M13 9PL, UK \\ 7 Observatory, PO Box 14, 00014 University of Helsinki, Finland \\ 8 W.W. Hansen Experimental Physics Laboratory, Stanford University, USA
}

Received 25 July 2007 / Accepted 29 February 2008

\section{ABSTRACT}

\begin{abstract}
Context. The solar magnetic activity and cycle are linked to an internal dynamo. Numerical simulations are an efficient and accurate tool to investigate such intricate dynamical processes.

Aims. We present the results of an international numerical benchmark study based on two-dimensional axisymmetric mean field solar dynamo models in spherical geometry. The purpose of this work is to provide reference cases that can be analyzed in detail and that can help in further development and validation of numerical codes that solve such kinematic problems.

Methods. The results of eight numerical codes solving the induction equation in the framework of mean field theory are compared for three increasingly computationally intensive models of the solar dynamo: an $\alpha \Omega$ dynamo with constant magnetic diffusivity, an $\alpha \Omega$ dynamo with magnetic diffusivity sharply varying with depth and an example of a flux-transport Babcock-Leighton dynamo which includes a non-local source term and one large single cell of meridional circulation per hemisphere. All cases include a realistic profile of differential rotation and thus a sharp tachocline.

Results. The most important finding of this study is that all codes agree quantitatively to within less than a percent for the $\alpha \Omega$ dynamo cases and within a few percent for the flux-transport case. Both the critical dynamo numbers for the onset of dynamo action and the corresponding cycle periods are reasonably well recovered by all codes. Detailed comparisons of butterfly diagrams and specific cuts of both toroidal and poloidal fields at given latitude and radius confirm the good quantitative agreement.

Conclusions. We believe that such a benchmark study will be a very useful tool since it provides detailed standard cases for comparison and reference.
\end{abstract}

Key words. Sun: magnetic fields - Sun: activity - Sun: interior - methods: numerical

\section{Introduction}

The Sun is an active star whose magnetism has a direct impact on the Earth and our technological society. Being able to understand and anticipate this magnetic activity is crucial and has thus been the subject of intense research. It is currently believed that the Sun operates an internal dynamo to generate, sustain and organize magnetic fields on both small and large scales. Given the complexity of the problem, i.e. the self-generation of magnetic fields by a turbulent rotating plasma and the non-linear interactions which yield this wide range of dynamical phenomena, numerical models of the magnetohydrodynamics of the Sun have been developed (Gilman 1983; Glatzmaier 1987; Cattaneo 1999; Brun et al. 2004). One class of such models is called mean field solar dynamo models and rely on the mean field theory developed mainly in the $1960 \mathrm{~s}$ and $1970 \mathrm{~s}$ (Steenbeck et al. 1966; Steenbeck \& Krause 1969; Roberts 1972; Stix 1976; Moffatt 1978; Krause \& Rädler 1980). In this framework, we seek to model the large scale mean field and use a simplified closure relationship in modeling the electromotive force (such as the $\alpha$-effect). Recently 2-D mean field models have made significant progress in modeling the characteristic magnetic features that the
Sun possesses, such as its butterfly diagram, its $11-y r$ cycle period, the phase relationship between poloidal and toroidal fields and the mainly dipolar polarity observed (Bonanno et al. 2002; Dikpati et al. 2004; Küker et al. 2001). They are even being used to predict the next solar cycle (cycle 24) (e.g. Dikpati \& Gilman 2006).

However, as of today, no systematic comparison of the numerical programs used by the various groups involved in understanding the solar dynamo and magnetism has been performed. We here propose to start such a comparison by defining a reference benchmark for the two dimensional solar dynamo problem. This benchmark is intended to be used by any scientist who wishes to calibrate and validate his or her code or to develop a new one. It is based on a set of simple and well-defined test cases, representative of what has been done in the kinematic approach of mean field theory so far. This benchmark is not intended to be exhaustive and to include a test case for all types of mean field dynamo models that have been performed over the last four decades. For instance, non-linear models of Malkus-Proctor type (see e.g. Brandenburg et al. 1991; Moss $\&$ Brooke 2000; Bushby 2006) will not be considered since we only want to focus on solving the induction equation and not the 
Navier-Stokes equation. We thus compute 2D axisymmetric mean field models of $\alpha \Omega$ and of flux-transport BabcockLeighton (BL) type (Babcock 1961; Leighton 1969; Wang et al. 1991; Choudhuri et al. 1995; Durney 1995; Dikpati \& Charbonneau 1999), in which we progressively introduce physical ingredients thought to play a key role in the solar dynamo. These ingredients are magnetic diffusivity sharply varying with depth, realistic large scale flows (differential rotation and meridional circulation) and a non-local source term for poloidal field. Moreover, we check the influence of imposing two different types of boundary conditions at the top of the domain. We choose here to study for these models the dynamo threshold and the temporal evolution of the magnetic field in cases called "critical cases" and we will present a more precise quantitative study of some cases in which the dynamo action is well-established, the "supercritical cases".

The paper is organized in the following manner. In Sect. 2, we present the equations, the initial and boundary conditions, the ingredients of the model and we present the test cases; Sect. 3 lists the numerical techniques used by the various groups to solve the induction equation. In Sect. 4, we discuss the results of our study and compare the solutions provided by the different codes and we conclude in Sect. 5.

\section{The solar dynamo model}

\subsection{Mean field equations}

To investigate the global solar cycle features produced by solar dynamo models, we start from the hydromagnetic induction equation, governing the evolution of the magnetic field $\boldsymbol{B}$ in response to advection by a flow field $\boldsymbol{U}$ and resistive dissipation due to the microscopic magnetic diffusivity $\eta_{\mathrm{m}}$.

$$
\frac{\partial \boldsymbol{B}}{\partial t}=\nabla \times(\boldsymbol{U} \times \boldsymbol{B})-\nabla \times\left(\eta_{\mathrm{m}} \nabla \times \boldsymbol{B}\right) .
$$

As we are working in the framework of mean field theory, we express both magnetic and velocity fields as the sum of large scale (that corresponds to the mean field) and small scale (associated with hydrodynamic turbulence) contributions. Averaging over some suitably chosen intermediate scales makes it possible to write two distinct induction equations for the mean and the fluctuating parts of the magnetic field. The mean field equation reads

$$
\frac{\partial\langle\boldsymbol{B}\rangle}{\partial t}=\nabla \times(\langle\boldsymbol{U}\rangle \times\langle\boldsymbol{B}\rangle)+\nabla \times\left\langle\boldsymbol{u}^{\prime} \times \boldsymbol{b}^{\prime}\right\rangle-\nabla \times\left(\eta_{\mathrm{m}} \nabla \times\langle\boldsymbol{B}\rangle\right),
$$

where $\langle\boldsymbol{B}\rangle$ and $\langle\boldsymbol{U}\rangle$ refer to the mean parts of the magnetic and velocity fields and $\boldsymbol{u}^{\prime}$ and $\boldsymbol{b}^{\prime}$ to the fluctuating components. A closure relation is then used to express the electromotive force $\left\langle\boldsymbol{u}^{\prime} \times \boldsymbol{b}^{\prime}\right\rangle$ in terms of the mean magnetic field $\langle\boldsymbol{B}\rangle$

$$
\begin{aligned}
\left\langle\boldsymbol{u}^{\prime} \times \boldsymbol{b}^{\prime}\right\rangle_{i} & =\alpha_{i j}\langle\boldsymbol{B}\rangle_{j}+\beta_{i j k} \frac{\partial\langle\boldsymbol{B}\rangle_{j}}{\partial x_{k}} \\
& =\alpha\langle\boldsymbol{B}\rangle_{i}-\beta(\nabla \times\langle\boldsymbol{B}\rangle)_{i} \quad \text { if isotropic. }
\end{aligned}
$$

This leads to the simplified mean field equation ${ }^{1}$

$$
\frac{\partial\langle\boldsymbol{B}\rangle}{\partial t}=\nabla \times(\langle\boldsymbol{U}\rangle \times\langle\boldsymbol{B}\rangle)+\nabla \times(\alpha\langle\boldsymbol{B}\rangle)-\nabla \times(\eta \nabla \times\langle\boldsymbol{B}\rangle),
$$

1 When they are isotropic, the pseudo-tensor $\alpha_{i j}=\alpha \delta_{i j}$ where $\delta_{i j}$ is the Kroenecker symbol and the tensor $\beta_{i j k}=\beta \epsilon_{i j k}$ where $\epsilon_{i j k}$ is the LeviCivita symbol. where $\eta=\eta_{\mathrm{m}}+\beta$ is now the effective magnetic diffusivity. For Babcock-Leighton models, a surface term $S \hat{\boldsymbol{e}}_{\phi}$ is used instead of the $\alpha$-effect term $\alpha\langle\boldsymbol{B}\rangle$ which is involved only in our $\alpha \Omega$ models.

Quantities will be considered henceforth as mean values so that we will omit the $\langle$.$\rangle . Working in spherical coordinates and$ under the assumption of axisymmetry, we write the total mean magnetic field $\boldsymbol{B}$ and the velocity field $\boldsymbol{U}$ as

$\boldsymbol{B}(r, \theta, t)=\boldsymbol{\nabla} \times\left(A_{\phi}(r, \theta, t) \hat{\boldsymbol{e}}_{\phi}\right)+B_{\phi}(r, \theta, t) \hat{\boldsymbol{e}}_{\phi}$,

$\boldsymbol{U}(r, \theta)=\boldsymbol{u}_{\mathrm{p}}(r, \theta)+r \sin \theta \Omega(r, \theta) \hat{\boldsymbol{e}}_{\phi}$.

We then introduce this poloidal/toroidal decomposition in Eq. (4). We obtain two coupled partial differential equations, one involving the poloidal potential $A_{\phi}$ and the other concerning the toroidal field $B_{\phi}$. In order to write these equations in a dimensionless form, we choose as the length scale the solar radius $R_{\odot}$ and as time scale the diffusion time $R_{\odot}^{2} / \eta_{\mathrm{t}}$ based on the turbulent diffusivity in the envelope $\eta_{\mathrm{t}}=10^{11} \mathrm{~cm}^{2} \mathrm{~s}^{-1}$,

$$
\begin{gathered}
\frac{\partial A_{\phi}}{\partial t}=\tilde{\eta} D^{2} A_{\phi}-R_{\mathrm{e}} \frac{\boldsymbol{u}_{\mathrm{p}}}{\varpi} \cdot \nabla\left(\varpi A_{\phi}\right)+C_{\alpha} \alpha B_{\phi}+C_{\mathrm{s}} S \\
\frac{\partial B_{\phi}}{\partial t}=\tilde{\eta} D^{2} B_{\phi}+\frac{1}{\varpi} \frac{\partial\left(\varpi B_{\phi}\right)}{\partial r} \frac{\partial \tilde{\eta}}{\partial r}-R_{\mathrm{e}} \varpi \boldsymbol{u}_{\mathrm{p}} \cdot \nabla\left(\frac{B_{\phi}}{\varpi}\right) \\
+C_{\Omega} \varpi\left(\nabla \times\left(A_{\phi} \hat{\boldsymbol{e}}_{\phi}\right)\right) \cdot \nabla \Omega,
\end{gathered}
$$

with $D^{2}=\left(\nabla^{2}-\frac{1}{\varpi^{2}}\right), \varpi=r \sin \theta$ and $\tilde{\eta}$ the normalized magnetic diffusivity.

Four adimensional numbers characterize the intensity of the various ingredients and enable all the quantities to be dimensionless. The intensity of the rotation $\Omega$ is characterized by $C_{\Omega}=\Omega_{\mathrm{eq}} R_{\odot}^{2} / \eta_{\mathrm{t}}$ where the rotation rate at the equator is $\Omega_{\mathrm{eq}} / 2 \pi=456 \mathrm{nHz}$. The intensity of the meridional circulation $u_{\mathrm{p}}$ is characterized by $R_{\mathrm{e}}=u_{0} R_{\odot} / \eta_{\mathrm{t}}$ where the peak amplitude of the meridional flow is $u_{0}=10 \mathrm{~ms}^{-1}$.

The $\alpha$-effect is characterized by $C_{\alpha}=\alpha_{0} R_{\odot} / \eta_{\mathrm{t}}$ and the BL source term by $C_{\mathrm{s}}=s_{0} R_{\odot} / \eta_{\mathrm{t}}$. In the critical cases, the intensity $\alpha_{0}$ of the $\alpha$-effect or $s_{0}$ of the BL source term are determined by looking for the threshold for dynamo action whereas in the supercritical cases, $\alpha_{0}$ and $s_{0}$ are fixed to a value about ten times higher than the threshold. Moreover, we note that an $\alpha$-effect is considered only for the regeneration of the poloidal field and not for the toroidal field so that we choose to study only $\alpha \Omega$ or Babcock-Leighton flux-transport dynamos.

\subsection{Initial and boundary conditions}

Equations (7) and (8) are solved in a segment of the meridional plane with the colatitude $\theta \in[0, \pi]$ and the normalized radius $r \in[0.65,1]$ i.e. from slightly below the tachocline (e.g. $r=0.7$ ) up to the solar surface. At $\theta=0$ and $\theta=\pi$ boundaries, we impose regularity conditions, i.e. both $A_{\phi}$ and $B_{\phi}$ are set to 0 . At $r=0.65$, we use a perfect conductor condition

$A_{\phi}=0$ and $\frac{\partial\left(r B_{\phi}\right)}{\partial r}=0$.

At the upper boundary, we can implement either of two different types of boundary conditions: we smoothly match our solution to an external potential field, i.e. we have a vacuum region for $r \geq 1$,

$B_{\phi}=0$ at $r=1$ and $D^{2} A_{\phi}=0$ for $r \geq 1$, 

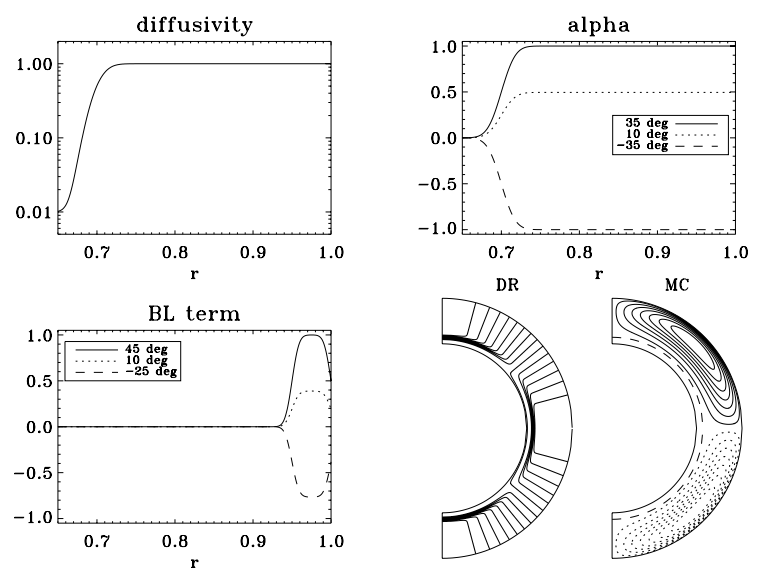

Fig. 1. The physical ingredients corresponding to Eqs. (13)-(15), (17), and (19). The angles indicated in the legends refer to the latitude. BL stands for Babcock-Leighton, DR for Differential Rotation and MC for Meridional Circulation.

or we set a pure radial field condition meaning

$B_{\phi}=B_{\theta}=0$ at $r=1$.

As initial conditions we use an arbitrary large scale field, that can be for instance a confined dipolar field, i.e.

$A_{\phi}=\frac{\sin \theta}{r^{2}}$ if $r \geq 0.7$ and $A_{\phi}=0$ otherwise and $B_{\phi}=0$.

\subsection{The physical ingredients}

The model "ingredients" are described below, shown in Fig. 1 and listed for each case in Table 1.

The rotation profile captures some realistic aspects of the Sun's angular velocity, deduced from helioseismic inversions, assuming a solid rotation below 0.66 and a differential rotation above the interface.

$\Omega(r, \theta)=\Omega_{\mathrm{c}}+\frac{1}{2}\left[1+\operatorname{erf}\left(\frac{r-r_{\mathrm{c}}}{d}\right)\right]\left(1-\Omega_{\mathrm{c}}-c_{2} \cos ^{2} \theta\right)$,

with $r_{\mathrm{c}}=0.7, d=0.02, \Omega_{\mathrm{c}}=0.92$ and $c_{2}=0.2$. With this profile, the radial shear is maximal at the tachocline.

We assume that the diffusivity in the envelope $\eta$ is dominated by its turbulent contribution whereas in the stable interior $\eta_{\mathrm{c}} \ll$ $\eta_{\mathrm{t}}$. We smoothly match the two different constant values with an error function which enables us to quickly and continuously transit from $\eta_{\mathrm{c}}$ to $\eta_{\mathrm{t}}$ i.e.

$\tilde{\eta}(r)=\frac{\eta_{\mathrm{c}}}{\eta_{\mathrm{t}}}+\frac{1}{2}\left(1-\frac{\eta_{\mathrm{c}}}{\eta_{\mathrm{t}}}\right)\left[1+\operatorname{erf}\left(\frac{r-r_{\mathrm{c}}}{d}\right)\right]$,

with $\eta_{\mathrm{c}}=10^{9} \mathrm{~cm}^{2} \mathrm{~s}^{-1}$.

The $\alpha$-effect is distributed in the whole convection zone (CZ), with a sharp variation with depth at the base of the CZ. It is antisymmetric with respect to the equator.

In this paper, two types of model are being studied. We have models in which we seek to evaluate the dynamo threshold and supercritical models for which we introduce a non-linear quenching in the source term of the poloidal field. The $\alpha$-effect profile will thus be different in these two cases.

In the models for which we investigate the thresholds for dynamo action, the profile of $\alpha$ is given by

$\alpha(r, \theta)=\frac{3 \sqrt{3}}{4} \sin ^{2} \theta \cos \theta\left[1+\operatorname{erf}\left(\frac{r-r_{\mathrm{c}}}{d}\right)\right]$, where the factor $3 \sqrt{3} / 4$ comes from normalization to unity. For the supercritical cases, since we are in a linear regime, the magnetic energy will grow exponentially with time and this will make it difficult to compare our results.

Thus for the supercritical cases, keeping all the other ingredients identical, we use a quenched form for $\alpha$, now timedependent, given by

$$
\begin{aligned}
\alpha(r, \theta, t)= & \frac{3 \sqrt{3}}{4} \sin ^{2} \theta \cos \theta\left[1+\operatorname{erf}\left(\frac{r-r_{\mathrm{c}}}{d}\right)\right] \\
& \times\left[1+\left(\frac{B_{\phi}(r, \theta, t)}{B_{0}}\right)^{2}\right]^{-1},
\end{aligned}
$$

with $B_{0}$ being an arbitrary normalization constant.

In Babcock-Leighton flux-transport dynamo models, the poloidal field owes its origin to the twist of magnetic loops emerging at the solar surface. Thus, the source has to be confined to a thin layer just below the surface and since the process is fundamentally non-local, the source term depends on the variation of $B_{\phi}$ at the base of the convection zone. For the critical cases, the expression is

$$
\begin{aligned}
S(r, \theta, t)= & \frac{1}{2}\left[1+\operatorname{erf}\left(\frac{r-r_{1}}{d_{1}}\right)\right]\left[1-\operatorname{erf}\left(\frac{r-1}{d_{1}}\right)\right] \\
& \times \cos \theta \sin \theta B_{\phi}\left(r_{c}, \theta, t\right) .
\end{aligned}
$$

For the supercritical cases, here too a quenching term is introduced to prevent the magnetic energy from growing exponentially without bound.

$$
\begin{aligned}
S(r, \theta, t)= & \frac{1}{2}\left[1+\operatorname{erf}\left(\frac{r-r_{1}}{d_{1}}\right)\right]\left[1-\operatorname{erf}\left(\frac{r-1}{d_{1}}\right)\right] \\
& \times\left[1+\left(\frac{B_{\phi}\left(r_{\mathrm{c}}, \theta, t\right)}{B_{0}}\right)^{2}\right]^{-1} \cos \theta \sin \theta B_{\phi}\left(r_{\mathrm{c}}, \theta, t\right)
\end{aligned}
$$

with $r_{1}=0.95, d_{1}=0.01$.

In these BL flux-transport dynamo models, meridional circulation is used to link the two sources of the magnetic field namely the base of the $\mathrm{CZ}$ and the solar surface. For its profile, we use a large single cell per hemisphere, directed poleward at the surface, vanishing at the bottom boundary $r=0.65$ and thus penetrating a little below the tachocline. We take a stream function

$\psi(r, \theta)=-\frac{2}{\pi} \frac{\left(r-r_{\mathrm{b}}\right)^{2}}{\left(1-r_{\mathrm{b}}\right)} \sin \left(\pi \frac{r-r_{\mathrm{b}}}{1-r_{\mathrm{b}}}\right) \cos \theta \sin \theta$,

which gives, through the relation $\boldsymbol{u}_{\mathrm{p}}=\nabla \times\left(\psi \hat{\boldsymbol{e}}_{\phi}\right)$, the following components of the meridional flow

$$
\begin{aligned}
u_{\mathrm{r}}= & -\frac{2\left(1-r_{\mathrm{b}}\right)}{\pi r} \frac{\left(r-r_{\mathrm{b}}\right)^{2}}{\left(1-r_{\mathrm{b}}\right)^{2}} \sin \left(\pi \frac{r-r_{\mathrm{b}}}{1-r_{\mathrm{b}}}\right)\left(3 \cos ^{2} \theta-1\right) \\
u_{\theta}= & {\left[\frac{3 r-r_{\mathrm{b}}}{1-r_{\mathrm{b}}} \sin \left(\pi \frac{r-r_{\mathrm{b}}}{1-r_{\mathrm{b}}}\right)+\frac{r \pi}{1-r_{\mathrm{b}}} \frac{\left(r-r_{\mathrm{b}}\right)}{\left(1-r_{\mathrm{b}}\right)} \cos \left(\pi \frac{r-r_{\mathrm{b}}}{1-r_{\mathrm{b}}}\right)\right] } \\
& \times \frac{2\left(1-r_{\mathrm{b}}\right)}{\pi r} \frac{\left(r-r_{\mathrm{b}}\right)}{\left(1-r_{\mathrm{b}}\right)} \cos \theta \sin \theta
\end{aligned}
$$

with $r_{\mathrm{b}}=0.65$. 
Table 1. Summary of the test cases, the cases followed by a prime have radial field boundary conditions (BC) at the top and the cases preceded by $S$ are supercritical (computed with a value of $C_{\alpha}$ or $C_{\mathrm{s}}$ well above the dynamo threshold).

\begin{tabular}{cccccccccc}
\hline \hline Case & $\Omega$-effect & Poloidal source term & Diffusivity & Meridional flow & $C_{\Omega}$ & $C_{\alpha}$ & $C_{\mathrm{s}}$ & $R_{\mathrm{e}}$ & Top BC \\
\hline $\mathrm{A}$ & Eq. (13) & $\alpha$ : Eq. (15) & $\tilde{\eta}=1\left(\eta_{\mathrm{c}}=\eta_{\mathrm{t}}\right.$ in Eq. (14)) & $\mathrm{NO}$ & $1.40 \times 10^{5}$ & $C_{\alpha}^{\text {crit }}(\mathrm{A})$ & 0 & 0 & Potential \\
$\mathrm{A}^{\prime}$ & Eq. (13) & $\alpha$ : Eq. (15) & $\tilde{\eta}=1\left(\eta_{\mathrm{c}}=\eta_{\mathrm{t}}\right.$ in Eq. (14)) & $\mathrm{NO}$ & $1.40 \times 10^{5}$ & $C_{\alpha}^{\text {crit }}\left(\mathrm{A}^{\prime}\right)$ & 0 & 0 & Radial \\
$\mathrm{SA}^{\prime}$ & Eq. (13) & $\alpha$ : Eq. (16) & $\tilde{\eta}=1\left(\eta_{\mathrm{c}}=\eta_{\mathrm{t}}\right.$ in Eq. (14)) & $\mathrm{NO}$ & $1.40 \times 10^{5}$ & 3.5 & 0 & 0 & Radial \\
$\mathrm{B}$ & Eq. (13) & $\alpha$ : Eq. (15) & Eq. (14) & $\mathrm{NO}$ & $1.40 \times 10^{5}$ & $C_{\alpha}^{\text {crit }}(\mathrm{B})$ & 0 & 0 & Potential \\
$\mathrm{B}^{\prime}$ & Eq. (13) & $\alpha$ : Eq. (15) & Eq. (14) & $\mathrm{NO}$ & $1.40 \times 10^{5}$ & $C_{\alpha}^{\text {crit }}\left(\mathrm{B}^{\prime}\right)$ & 0 & 0 & Radial \\
$\mathrm{SB}^{\prime}$ & Eq. (13) & $\alpha$ : Eq. (16) & Eq. (14) & NO & $1.40 \times 10^{5}$ & 3.5 & 0 & 0 & Radial \\
C & Eq. (13) & BL: Eq. (17) & Eq. (14) & Eq. (19) & $1.40 \times 10^{5}$ & 0 & $C_{\mathrm{s}}^{\text {crit }}(\mathrm{C})$ & 700 Potential \\
$\mathrm{C}^{\prime}$ & Eq. (13) & BL: Eq. (17) & Eq. (14) & Eq. (19) & $1.40 \times 10^{5}$ & 0 & $C_{\mathrm{s}}^{\text {crit }}\left(\mathrm{C}^{\prime}\right)$ & 700 & Radial \\
SC $^{\prime}$ & Eq. (13) & BL: Eq. (18) & Eq. (14) & Eq. (19) & $1.40 \times 10^{5}$ & 0 & 35 & 700 & Radial \\
\hline
\end{tabular}

\subsection{Description of the test cases}

Cases $\mathrm{A}$ and $\mathrm{B}$ are 2 different cases of pure $\alpha \Omega$ dynamos, all using the conical differential rotation profile (13) with a sharp tachocline and an $\alpha$-effect distributed in the whole convection zone. Case A involves a constant diffusivity whereas in cases B and $\mathrm{C}$, we introduce a diffusivity gradient between the core and the envelope.

Case $\mathrm{C}$ is a Babcock-Leighton flux-transport dynamo, in which the source term for the poloidal field is due to the twisted nature of active regions observed at the solar surface. The meridional circulation (MC) is introduced as one large single cell directed poleward at the surface, in accordance with solar observations. In this model, we keep the magnetic diffusivity gradient and a solar-like differential rotation.

Cases A, B, C ( $\left.\mathrm{A}^{\prime}, \mathrm{B}^{\prime}, \mathrm{C}^{\prime}\right)$ are computed with potential (vertical) field boundary conditions at the surface. Introducing these two types of boundary condition will enable researchers who are new to the field to test their codes with BCs whose implementation can demand careful work.

In addition to these cases seeking to assess the dynamo threshold, we perform a more detailed comparison of the magnetic field behaviour when the dynamo number has a value well above the dynamo threshold. These computations are also performed with radial field boundary conditions at the surface and with a fixed resolution of $101 \times 101$ grid points. Since these cases are the supercritical cases of $\mathrm{A}^{\prime}, \mathrm{B}^{\prime}$ and $\mathrm{C}^{\prime}$, they are denoted as $\mathrm{SA}^{\prime}, \mathrm{SB}^{\prime}$ and $\mathrm{SC}^{\prime}$.

\section{The codes involved}

Eight different codes based on various numerical techniques are used to solve the mean field induction equation presented in the preceding section. The main methods are finite differences, finite elements and spectral decomposition. This enables this benchmark to compare very different techniques representative of what has been done as of today in the community to solve Eqs. (7) and (8). The codes are described briefly in this section and detailed descriptions can be found in the references given.

\subsection{STELEM code}

The STELEM (STellar ELEMemts) code uses a finite element method in space and a third order scheme in time (Burnett 1987; Jouve \& Brun 2007). The code solves Eqs. (7) and (8) by seeking the approximate solutions $\tilde{A}_{\phi}$ and $\tilde{B}_{\phi}$ as linear combinations of trial functions $\zeta_{i}(r, \theta)$ (Lagrange interpolating polynomials of degree 1 associated with the grid points (linear functions) for 1st order interpolation). The coefficients of the two linear combinations are dependent on time only.

The main steps of the method are the following:

- Our domain (defined by $r_{\mathrm{b}} \leq r \leq 1,0 \leq \theta \leq \pi$ ) is divided into smaller regions called elements. These elements are quadrilaterals with a node at each corner when the trial functions are 1st order Lagrange polynomials.

- In each element, the PDEs are transformed into ordinary differential equations in time involving the coefficients of the linear combinations.

- The terms in the element equations are numerically evaluated for each element in the mesh. The resulting numbers are assembled into a much larger set of equations called the system equations.

- The boundary conditions are taken into account. They are implemented here as Dirichlet type conditions: the top boundary condition is a potential field or purely radial field. We assume a perfectly conducting bottom boundary.

The temporal scheme that we use is adapted from Spalart et al. (1991). The 3 steps of this explicit scheme enable us to get an error as small as $O\left(\Delta t^{3}\right)$ (see Jouve \& Brun 2007; Charbonneau 2005).

\subsection{NDYND code}

NDYND is a nonlinear dynamo code with density evolution (the density does not evolve in our present calculations) (Brandenburg et al. 1992). It is an explicit code that solves the mean field dynamo equations on a uniform mesh in $r$ and $\theta$ to second order. Advection-type terms are solved to fourth order. For mesh points on the boundaries, one-sided second order derivative formulae are used whilst on the axis appropriate symmetry conditions are used. Following Proctor (1977), the equations are stepped forward in time using a second order DufortFrankel scheme that treats the diffusion operator semi-implicitly. The potential field boundary condition is implemented by calculating the value of $A_{\phi}$ on the boundary through matrix multiplication in terms of the values of two mesh points inside the domain (Jepps 1975). The matrices are calculated prior to the simulation for a given mesh. For case $\mathrm{C}$ described below, the value of $B_{\phi}(r=0.7)$ is obtained by linear interpolation between two neighbouring mesh points.

\subsection{MBRK (Moss Brooke Runge-Kutta) code}

This code integrates the dynamo equations over $r_{\mathrm{b}} \leq r \leq 1$, $0 \leq \theta \leq \pi$, on a uniform 2-D grid. Spatial derivatives are second order, and time-stepping is by a second order Runge-Kutta 
method (in tests, a fourth order Runge-Kutta scheme gave indistinguishable results). When vacuum boundary conditions are used at the outer boundary, $r=1$, a non-local matrix multiplication method is used (as in NDYND above). On other boundaries, when needed, derivatives on the boundaries are evaluated by their one-sided second order representations. In case $C$, the coefficient $B_{\phi}\left(r_{\mathrm{c}}, \theta\right)$ occurring in the source term is obtained by linear interpolation from the neighbouring values. The code is basically that of Moss \& Brooke (2000).

\subsection{MEFISTO (MEan Fleld STellar simulatiOn) code}

The MEFISTO code solves the induction equation in a spherical shell from $r_{\mathrm{b}}$ to 1 and $0 \leq \theta \leq \pi$ in radius and latitude, respectively (Käpylä et al. 2006). The grid is uniformly spaced and spatial derivatives are computed using explicit second order accurate finite differences. As time-stepping methods, either firstorder Euler, or second order Adams-Bashforth schemes can be used. The boundary conditions are implemented using one-sided expressions of the first derivatives to yield the boundary value. For case $\mathrm{C}$ described above, the value of $B_{\phi}\left(r_{\mathrm{c}}, \theta\right)$ is obtained from linear interpolation between the adjacent grid points.

\subsection{HAO Dynamo code1}

The dynamo code of M. Rempel uses a fully explicit finite difference scheme, which is second order in time and space (the advection terms are discretized using a MacCormack scheme). In its current version the code only supports a vertical field boundary condition at the surface. The code has been used recently by Rempel (2006) to study non-linear Lorentz-force feedback on differential rotation and meridional flow. For the purpose of this benchmark study, the non-linear terms were switched off and the code was used in a purely kinematic way. The results presented in this paper were computed in only one hemisphere with symmetry imposed through the equatorial boundary condition.

\subsection{HAO Dynamo code2}

The dynamo code of M. Dikpati numerically solves the two coupled partial differential equations of advection-diffusion type by using a semi-implicit scheme, namely the Peaceman-RachfordAlternating-Direction-Implicit scheme (Ames 1992; Press et al. 1992). Writing the equations in operator notation as

$\frac{\partial B_{\phi}}{\partial t}=\left[L_{\mathrm{r}}+L_{\theta}\right] B_{\phi}+S_{1}$

$\frac{\partial A}{\partial t}=\left[L_{\mathrm{r}}+L_{\theta}\right] A+S_{2}$,

in which $L_{\mathrm{r}}$ contains all the operators in $r$ and $L_{\theta}$ in $\theta$ and $S_{1}$ and $S_{2}$ denote the cross-terms, the first half of the time-step is advanced by treating the $r$-direction explicitly and $\theta$-direction implicitly, the next half time-step in the reverse manner. The time-step is determined by satisfying the CFL condition. Since the diffusive terms are parabolic, whereas the advective terms are hyperbolic, a space-centered finite differencing scheme for the diffusive terms and a Lax-Wendroff scheme for the advective terms have been applied in order to maintain the second order accuracy in these mixed systems (see Dikpati 1996; and Dikpati \& Charbonneau 1999 for more details regarding the calculations of the tridiagonal elements, initialization and boundary conditions).

\subsection{CTDYN (Catania Dynamo) code}

The dynamo code used for the benchmark is a pseudospectral eigenvalue code.

The induction equation is solved with a finite-difference scheme for the radial dependence and a Legendre polynomial expansion for the angular dependence. In particular, the following expansions for the field is used

$\hat{A}(x, \theta)=\mathrm{e}^{\lambda t} \sum_{n} a_{n}(x) P_{n}^{(1)}(\cos \theta)$,
$\hat{B}(x, \theta)=\mathrm{e}^{\lambda t} \sum_{m} b_{m}(x) P_{m}^{(1)}(\cos \theta)$,

where $\lambda$ is the (complex) eigenvalue, $n=1,3,5, \ldots$ and $m=$ $2,4,6, \ldots$ for antisymmetric modes, and $n \leftrightarrow m$ for symmetric modes. Vacuum boundary conditions at the surface and perfectly conducting conditions at $x=x_{\mathrm{i}}=0.65$ are then translated into simple ordinary differential equations involving the coefficients $a_{n}$ and $b_{m}$.

By substituting (24) and (25) in the induction equation, an infinite set of ODEs is obtained, that can be conveniently truncated in $n$ when the desired accuracy is achieved. The system is in fact solved by means of a second order accurate finite difference scheme and the basic computational task is thus to numerically compute eigenvalues and eigenvectors of a block-band diagonal real matrix of dimension $M \times n, M$ being the number of mesh points and $n$ the number of harmonics, $M(\alpha) v=\lambda v$ and $v$ is in general a complex eigenvector.

This basic algorithm is embedded in a bisection procedure in order to determine the critical dynamo number needed to find a purely oscillatory solution, for which $\mathfrak{R} e(\lambda)=0$ Numerically a zero is accepted when the dimensionless quantity $\mathfrak{R} e(\lambda) R_{\odot}^{2} / \eta_{\mathrm{t}}$ is no greater than $10^{-3}$ in the following calculations. Greater accuracy usually requires a more refined spatial grid; see Bonanno (2004) for further details about the code.

\subsection{HOLLERBACH code}

The MHD code developed by Hollerbach (2000) is an incompressible, spherical, spectral scheme which solves the momentum equation, the induction equation, and the temperature equation in the Boussinesq approximation. The dynamo benchmark performed by R. Arlt employs only the induction equation. Full MHD computations of the magnetorotational instability with this code are given in Arlt et al. (2003).

The magnetic field is decomposed into two scalar potentials, $g$ and $h$, defined by

$\boldsymbol{B}=\nabla \times\left(g \hat{\boldsymbol{e}}_{\mathrm{r}}\right)+\nabla \times \nabla \times\left(h \hat{\boldsymbol{e}}_{\mathrm{r}}\right)$,

where $\hat{\boldsymbol{e}}_{\mathrm{r}}$ is the radial unit vector. The potentials depend on $r$, $\theta$, and $\phi$. The actual equations being solved are the radial component of the induction equation and the radial component of the curled induction equation. The potentials $g$ and $h$ are used in their spectral representation with spherical harmonics for the azimuthal and latitudinal structure, such that

$g(r, \theta, \phi)=\sum_{m=0}^{ \pm M} \sum_{l=m^{\prime}}^{L} g_{l m}(r, t) P_{l}^{|m|}(\cos \theta) \mathrm{e}^{\mathrm{i} m \phi}$,

where $m^{\prime}=\max (m, 1)$ and $L$ and $M$ are the truncations of the spectral expansion. In this paper, we set $M=0$ for all computations because of axisymmetry. Finally, these expressions 
are reintroduced in the induction equation which is integrated numerically.

The radial dependence of $g$ and $h$ is further expanded in Chebyshev polynomials which have a high density of zeros near the radial boundaries, hence providing very good resolution in the boundary layers. Two additional modes are used to implement the radial boundary conditions for the magnetic field. The physical condition for a boundary with vacuum is $\nabla \times \boldsymbol{B}=0$ for the exterior and leads to particular conditions for $g$ and $h$ and likewise for the conditions for perfectly conducting boundaries.

The diffusive part of the system is solved implicitly. The time-stepping is a Runge-Kutta integration with a second order predictor and corrector steps. While the determination of critical dynamo numbers of $\alpha$-type dynamos is a linear problem, we have kept the scheme in order to be able to add nonlinearities such as quenching functions or nonlocality to the source term $S$.

The non-linear routine consists of a transformation of the spectral potentials into a real-space $\boldsymbol{B}$, the computation of $\boldsymbol{U} \times \boldsymbol{B}+$ $S$, and the curling and back-transformation into spectral space. The velocity field $\boldsymbol{U}$ and the source term $S$ are only defined in real space.

\section{Results}

The goal of this benchmark is to publish a detailed analysis of the results obtained by 8 different codes running on well-defined test cases and to quantitatively assess the agreement between the codes. It aims at providing the community with precise standard solar dynamo cases to which to refer. In order to compare our results both qualitatively and quantitatively, we indicate in tables for each case the critical dynamo number $C_{\alpha}^{\text {crit }}$ above which the solution is growing exponentially, and the corresponding frequency $\omega$, defined by $\omega=2 \pi / T$ with the magnetic cycle pe$\operatorname{riod} T$ (i.e. twice the period of the sunspot cycle) in terms of the diffusive time $R_{\odot}^{2} / \eta_{\mathrm{t}}$. In addition to these quantitative results, we provide the butterfly diagrams and the evolution of the field lines in a meridional plane for each case, in order to follow the behaviour of the magnetic field configuration over time. For the supercritical cases, since the intensity of the magnetic field is saturated through the quenching term, we can perform a detailed comparison of the field intensity at selected points in the computational domain during a part of the cycle. We can then see the deviation of each curve obtained by the different codes from the mean curve, which will be considered to be the "optimal solution". Finally, to confirm that all codes converge to the same solution, we run convergence tests for 2 particular cases $\left(B\right.$ and $\left.C^{\prime}\right)$, which allow us to follow the evolution of the values of $C_{\alpha}$ or $C_{\mathrm{s}}$ and $\omega$ as the spatial resolution is increased.

\subsection{Case $A$}

\subsubsection{Critical cases}

Table 2 shows the results for the critical dynamo numbers and periods for cases $\mathrm{A}$ and $\mathrm{A}^{\prime}$. The results are in good agreement with each other, the relative standard deviation reaching only the value of 0.006 for $C_{\alpha}$ in both cases, meaning that the values are all gathering close to the mean. The agreement on the period is also quite satisfactory, the relative standard deviation being again of the order of a few parts in a thousand.

In these cases, we find that only relatively low spatial resolution is needed for convergence and close agreement. Even though the error function representations of $\alpha$ and $\Omega$ imply relatively rapid changes in these functions, they are smooth and
Table 2. Critical values of dynamo numbers and frequencies for case A (with potential boundary conditions) and case $\mathrm{A}^{\prime}$ (with radial field conditions) with the spatial and temporal resolution for each code. We indicate the mean value, the standard deviation and the relative standard deviation (R.S.D) (standard deviation divided by the mean value) for each case. Asterisks indicate particular cases: for HOLLERBACH, the resolution is given in terms of the number of spectral modes and for CTDYN in terms of grid points in $r$ and spectral modes in $\theta$. CTDYN does not evolve the system in time, which is why we do not indicate any time-step for this code.

\begin{tabular}{cccccc}
\hline \hline Case & Code & Resolution & $\Delta t$ & $C_{\alpha}^{\text {crit }}$ & $\omega$ \\
\hline A & STELEM & $65 \times 65$ & $10^{-5}$ & 0.385 & 157 \\
A & NDYND & $81 \times 81$ & $5 \times 10^{-6}$ & 0.385 & 158 \\
A & MBRK & $81 \times 81$ & $5 \times 10^{-6}$ & 0.390 & 159 \\
A & CTDYN & $70 \times 70 *$ & - & 0.388 & 160 \\
A & HAO2 & $101 \times 101$ & $10^{-5}$ & 0.388 & 156 \\
A & HOLLER & $60 \times 60 *$ & $5 \times 10^{-5}$ & 0.385 & 159 \\
Mean val & & & & $\mathbf{0 . 3 8 7}$ & $\mathbf{1 5 8 . 1}$ \\
Std Dev. & & & & $\mathbf{0 . 0 0 2}$ & $\mathbf{1 . 4 7 2}$ \\
R. S. D. & & & & $\mathbf{0 . 0 0 6}$ & $\mathbf{0 . 0 0 9}$ \\
\hline A $^{\prime}$ & STELEM & $65 \times 65$ & $10^{-5}$ & 0.366 & 158 \\
A $^{\prime}$ & NDYND & $81 \times 81$ & $5 \times 10^{-6}$ & 0.369 & 156 \\
A $^{\prime}$ & MBRK & $81 \times 81$ & $5 \times 10^{-6}$ & 0.372 & 158 \\
A $^{\prime}$ & MEFISTO & $121 \times 121$ & $10^{-6}$ & 0.368 & 158 \\
A $^{\prime}$ & HAO1 & $128 \times 128$ & $3 \times 10^{-6}$ & 0.368 & 157 \\
Mean val & & & & $\mathbf{0 . 3 6 9}$ & $\mathbf{1 5 7 . 4}$ \\
Std Dev. & & & & $\mathbf{0 . 0 0 2}$ & $\mathbf{0 . 8 9 4}$ \\
R.S. D. & & & & $\mathbf{0 . 0 0 6}$ & $\mathbf{0 . 0 0 6}$ \\
\hline
\end{tabular}

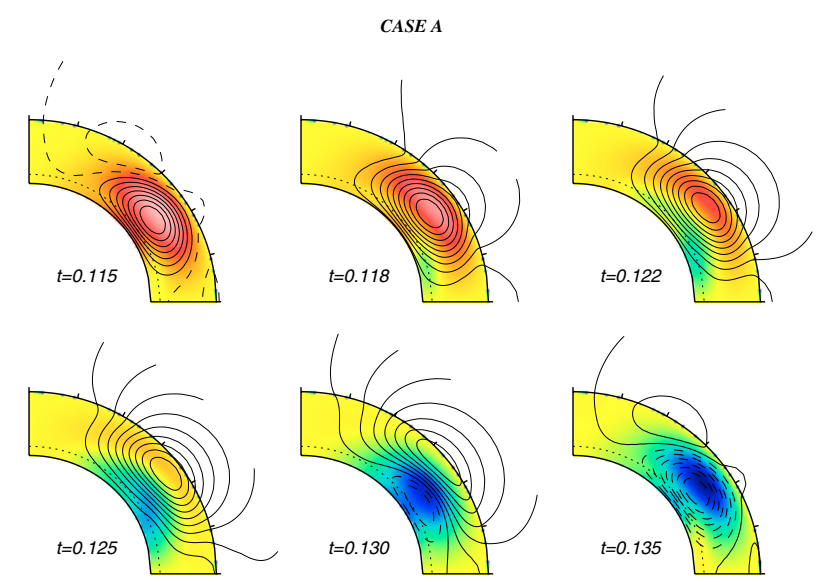

Fig. 2. Results for case A: pure $\alpha \Omega$ dynamo with constant magnetic diffusivity. The results are shown for $C_{\alpha}=C_{\alpha}^{\text {crit }}$. The figure shows the evolution of the contours of the poloidal potential (left panel) and of the toroidal field (right panel) during half a magnetic cycle. Red (blue) colours indicate positive (negative) toroidal field and plain (dotted) lines indicate clockwise (anticlockwise) poloidal field lines.

the models do not produce strong radial or latitudinal gradients in the induced toroidal or poloidal fields. Instead, the magnetic fields are organized in very large structures that smoothly match the boundary conditions. Since these solutions occur for dynamo numbers only slightly above critical, no strong gradients in toroidal or poloidal fields should arise at later times either. We see that the solutions satisfying radial field boundary conditions at $r=1$ are excited at lower dynamo numbers than for the potential field boundary conditions. But the dynamo frequency is nearly the same for both boundary conditions, about 158 (corresponding to a period of 30.9 years in dimensional units, compared to 11 years for the solar cycle). Figures 2 and 3 show the 


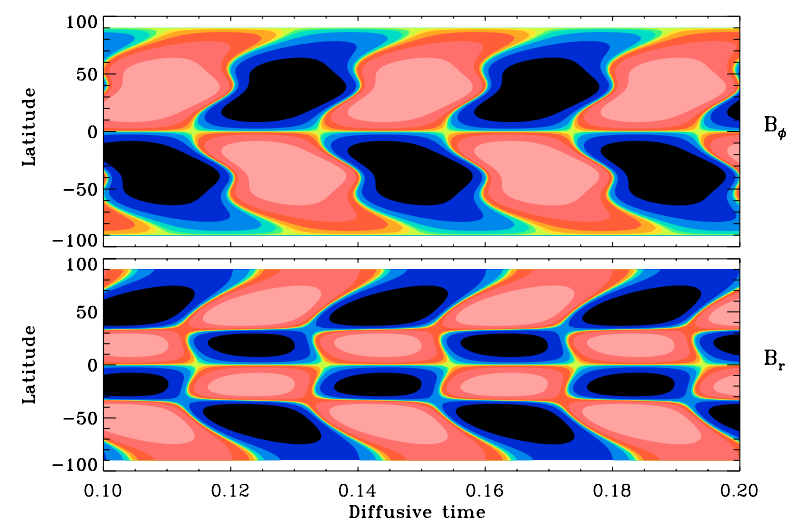

Fig. 3. Case A: butterfly diagram i.e. time-latitude cut of the toroidal field at $0.7 R_{\odot}$ (upper panel) and of the radial field at the surface (lower panel). Red (blue) colours indicate positive (negative) values of the field.

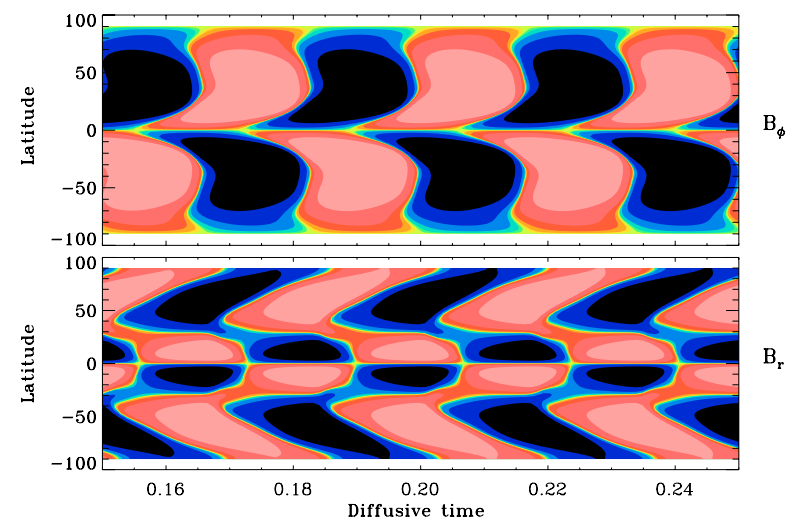

Fig. 4. Same as case A but for case $\mathrm{SA}^{\prime}$.

behaviour of the solution over time. Figure 2 shows the evolution of the field lines in the meridional plane in the northern hemisphere during half a magnetic cycle and Fig. 3 shows a timelatitude cut of the toroidal field at the base of the convection zone $(r=0.7)$ and of the radial field at the surface in both hemispheres. In this case the solution produces a butterfly diagram whose "wings" for the toroidal field slope toward the poles with increasing time, opposite to the solar case.

\subsubsection{Supercritical case}

We have previously discussed models $\mathrm{A}$ and $\mathrm{A}^{\prime}$ in the case where $C_{\alpha}$ was at its critical value, i.e. in the case of a quasi-stationary magnetic energy. We now compare the various codes for a supercritical case of model $\mathrm{A}^{\prime}$ (with vertical field conditions at the surface). We thus choose for this case a value of $C_{\alpha}$ of 3.5, almost 10 times the critical value of $C_{\alpha}$ in this case.

Figure 4 represents the butterfly diagram obtained in case $\mathrm{SA}^{\prime}$. The evolution of the magnetic field is close to what we found for the critical case. We can nevertheless notice that the high latitude polar branch is reduced in this case compared to case A but, in the latitudinal band where the sunspots appear, we again see that the toroidal field is moving poleward, contrary to what is observed in the Sun. We also see that increasing the $C_{\alpha}$ decreases the cycle period in this case.

Figure 5 shows the deviation from the mean value of cuts at various radii and latitudes of the toroidal and radial fields in the magnetic energy-saturated regime for the different codes. All curves were adjusted so that $B_{\phi}$ at the base of the convection
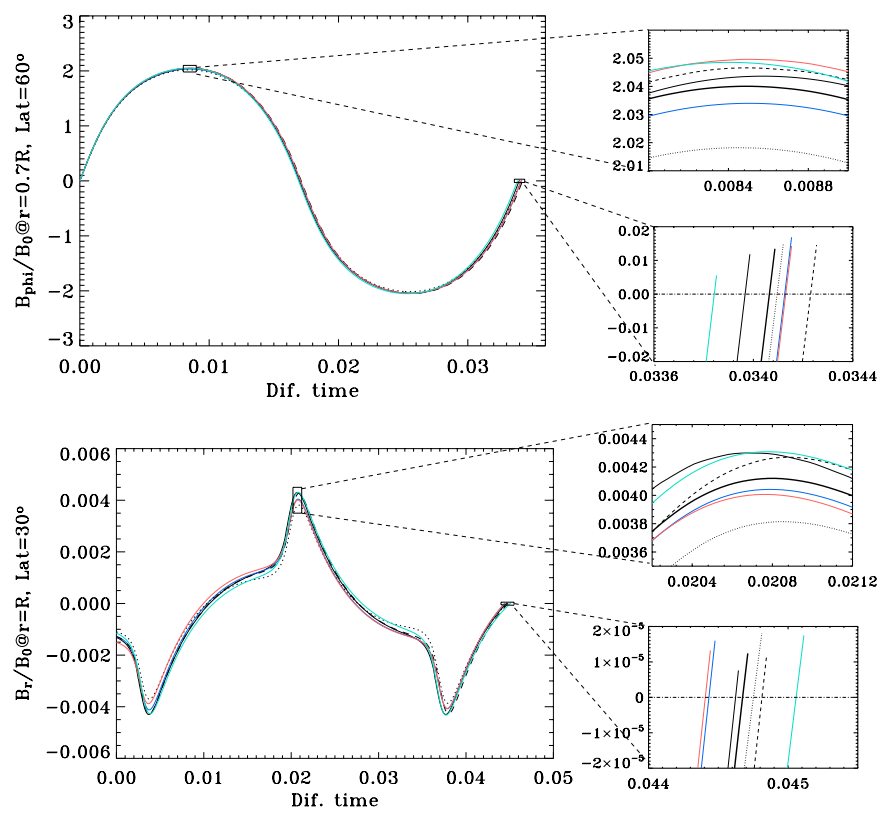

Fig. 5. Case $\mathrm{SA}^{\prime}$ : comparison between the values of $B_{\phi}(r=$ 0.7 , latitude $\left.=60^{\circ}\right)$ and $B_{\mathrm{r}}\left(r=1\right.$, latitude $\left.=30^{\circ}\right)$ normalized by $B_{0}$ obtained by the different codes during a cycle. The right panels show a zoom on the first maximum of the field and on the first time the field returns to zero after one cycle period. The thick line represents the mean value of the field and the coloured lines represent the curves obtained by the different codes. The colour coding is the following: HAO1 in blue, MBRK in red, STELEM in green, NDYND in black dashed, HAO2 in black and MEFISTO in black dotted.

zone is exactly 0 at $t=0$. We show for each curve a zoom on the first maximum of $B_{\phi}$ at the base of the convection zone and of $B_{\mathrm{r}}$ at the solar surface and on the crossing of zero after one cycle period. This procedure enables us to compare both the amplitude shift and the phase shift caused by the various codes.

We first see that the deviations between the codes are more significant for $B_{\mathrm{r}}$ at the surface. This could be due to various effects such as the imposition of the boundary conditions or the sharper profile of $B_{\mathrm{r}}$ close to its maximum compared to $B_{\phi}$. Nevertheless, the deviation from the mean curve stays under $7 \%$ of the maximum value of the field and this deviation drops to about $1.2 \%$ for $B_{\phi}$. The agreement on the field maxima is thus very satisfactory.

Looking at the instant where $B_{\phi}$ and $B_{\mathrm{r}}$ return to zero after one period enables us to see the significance of the phase shift between the various solutions. Again the differences are more pronounced for the curves for the radial field. The curves all cross zero in the very small time interval [0.0444, 0.0450] which is about $1.6 \%$ of the cycle period. The length of this time interval is the same for the curves for the toroidal field.

This pure $\alpha \Omega$ dynamo model includes a constant magnetic diffusivity. This ingredient of the model is one of the most poorly known but we can reasonably assume that the net diffusivity should be much lower in the convection-free radiative core than in the turbulent envelope and thus that the diffusivity should vary with depth in our computational domain. In the following case, we refine our model to test the influence of applying a gradient of magnetic diffusivity in a pure $\alpha \Omega$ model and how the various codes cope with this more challenging computation. 
Table 3. Same as for Table 2, for cases B and B'.

\begin{tabular}{cccccc}
\hline \hline Case & Code & Resolution & $\Delta t$ & $C_{\alpha}^{\text {crit }}$ & $\omega$ \\
\hline B & STELEM & $129 \times 129$ & $10^{-6}$ & 0.410 & 172 \\
B & NDYND & $81 \times 81$ & $10^{-5}$ & 0.405 & 172 \\
B & MBRK & $128 \times 128$ & $10^{-6}$ & 0.411 & 172 \\
B & CTDYN & $70 \times 70 *$ & - & 0.411 & 172 \\
B & HAO2 & $101 \times 101$ & $10^{-5}$ & 0.403 & 171 \\
B & HOLLER & $60 \times 60 *$ & $5 \times 10^{-5}$ & 0.408 & 173 \\
Mean val & & & & $\mathbf{0 . 4 0 8}$ & $\mathbf{1 7 2}$ \\
Std Dev. & & & & $\mathbf{0 . 0 0 3}$ & $\mathbf{0 . 6 3 2}$ \\
R. S. D. & & & & $\mathbf{0 . 0 0 8}$ & $\mathbf{0 . 0 0 4}$ \\
\hline B' & STELEM & $65 \times 65$ & $10^{-5}$ & 0.387 & 169 \\
B $^{\prime}$ & NDYND & $81 \times 81$ & $10^{-5}$ & 0.385 & 168 \\
B' & MBRK & $128 \times 128$ & $10^{-6}$ & 0.391 & 169 \\
B $^{\prime}$ & MEFISTO & $121 \times 121$ & $10^{-6}$ & 0.387 & 169 \\
B' & HAO1 & $128 \times 128$ & $3 \times 10^{-6}$ & 0.387 & 169 \\
Mean val & & & & $\mathbf{0 . 3 8 7}$ & $\mathbf{1 6 8 . 8}$ \\
Std Dev. & & & & $\mathbf{0 . 0 0 2}$ & $\mathbf{0 . 4 4 7}$ \\
R. S. D. & & & & $\mathbf{0 . 0 0 6}$ & $\mathbf{0 . 0 0 3}$ \\
\hline
\end{tabular}

\subsection{Case B}

In this case, the magnetic diffusivity is no longer constant, as indicated by Eq. (14). This refinement enables us to test how the various codes cope with a non constant diffusivity profile in the model. Here again, the comparison is made by looking at the critical $C_{\alpha}$, at the periods, and at the strength of the magnetic field during one cycle.

\subsubsection{Critical cases}

As Table 3 shows, the agreement of all codes on the critical values of $C_{\alpha}$ is quite good, even at low resolution. We note that the diffusivity gradient makes it more difficult for the dynamo to be excited and produces shorter cycles (see Table 2 for a close comparison with case A), indeed the frequency is now close to 170 , which corresponds to a sunspot period of 28.7 years, given our choice of $\eta_{\mathrm{t}}$.

We note again that in this case the dynamo is more easily excited when radial BCs are imposed, and that the period of the dynamo waves is slightly increased in case $\mathrm{B}^{\prime}$. Moreover, the agreement on the value of the critical dynamo number is better in case $\mathrm{B}^{\prime}$, showing that the imposition of potential boundary conditions can induce some divergence between codes. We then conclude that, even in this simple $\alpha \Omega$ model the choice and implementation of boundary conditions can already be a delicate issue.

Figure 6 indicates the convergence behaviour of each code toward the values quoted in Table 3 for case $B$ with potential BCs. Note that for each quantity $\left(C_{\alpha}^{\text {crit }}\right.$ and $\left.\omega\right)$, the spread of the values in the converged part of the diagram is less than $1 \%$ of the absolute value. That is, all results that fall in the diagram agree within better than $1 \%$, as we can already see in Table 3 . All results seem to converge approximately towards the same point, especially for the frequency, where the agreement is extremely good. We note that in this case, most codes have already converged with a resolution of $80 \times 80$, confirming that this calculation does not require a very high resolution in spite of the sharp diffusivity profile and the imposition of potential boundary conditions. We note that the convergence is very fast for the pseudospectral code CTDYN, since the values at a resolution of $40 \times 40$ are already close to the converged value.
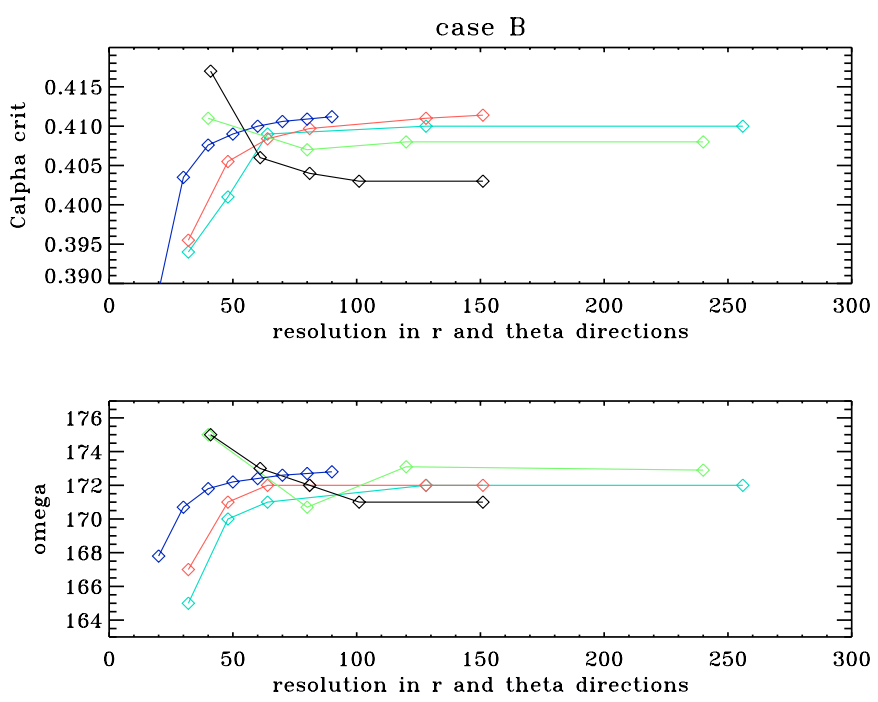

Fig. 6. Convergence test for case B: evolution of the values of $C_{\alpha}^{\text {crit }}$ and $\omega$ as functions of the spatial resolution. The colour coding is the following: HAO2 in black, CTDYN in blue, STELEM in green, MBRK in red, HOLLER in light green for which the resolution is the number of collocation points, twice the number of Chebyshev polynomials.
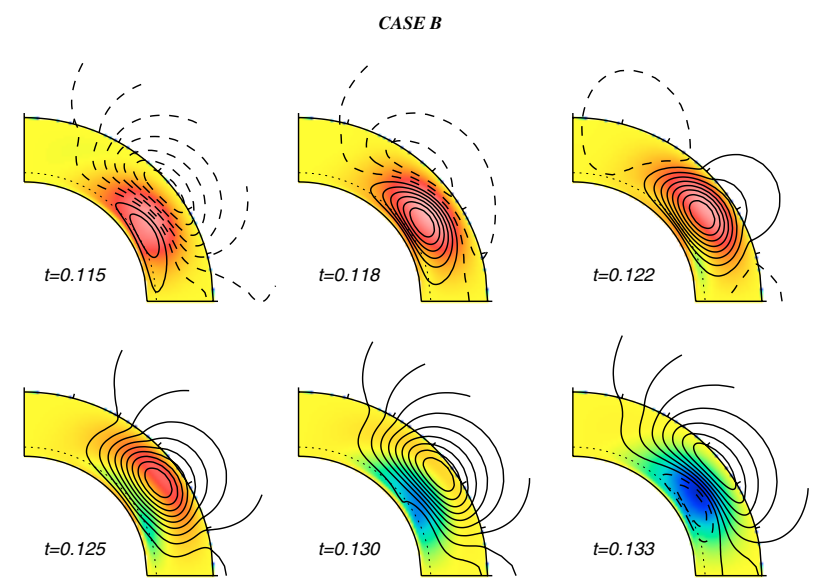

Fig. 7. Same as Fig. 2 but for case B: pure $\alpha \Omega$ dynamo with a jump of magnetic diffusivity from $0.01 \eta_{\mathrm{t}}$ below the tachocline to $\eta_{\mathrm{t}}$ in the convection zone. The results are shown for $C_{\alpha}=C_{\alpha}^{\text {crit }}$.

As we can see in Figs. 7 and 8, the magnetic field behaviour is close to what was found in the constant diffusivity case (case A) except that the poloidal field lines seem to be less diffuse in the tachocline due to the presence of the sharp diffusivity gradient in this zone. In the meridional plane, the regions of generation and destruction of the magnetic structures are the same and the directions of rise and migration of the field are identical to case A. However, we notice in the butterfly diagram that the regions of strongest magnetic intensity are confined in a much smaller region around $30^{\circ}$ latitude approximately in the region of strongest $\alpha$-effect. The polar branch at high latitudes (above $60^{\circ}$ ) of the toroidal field is more stretched in time and less intense compared to the regions of strongest intensity than in case A. The addition of variable diffusivity and thus of diffusivity gradients have some impact on the field location and its organization in finer structures. 


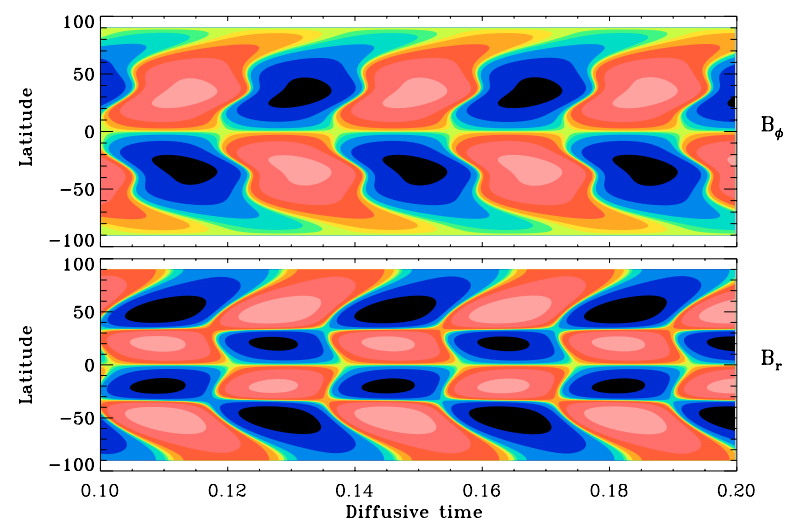

Fig. 8. Same as Fig. 3 but for case B.

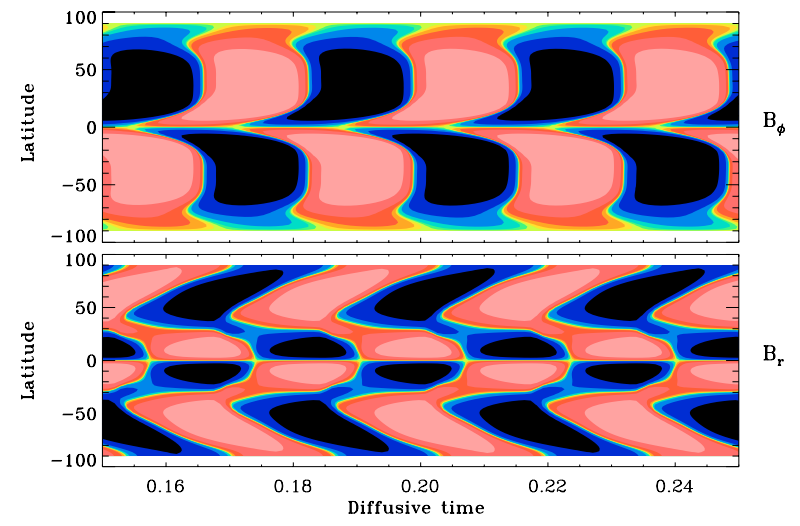

Fig. 9. Same as Fig. 4 but for case $\mathrm{SB}^{\prime}$ : supercritical case of pure $\alpha \Omega$ dynamo with variable diffusivity: butterfly diagram i.e. time-latitude cut of the toroidal field at 0.7 (upper panel) and of the radial field at the surface (lower panel).

\subsubsection{Supercritical case}

Figure 9 shows the butterfly diagram obtained in case $\mathrm{SB}^{\prime}$. It is again very similar to case $\mathrm{SA}^{\prime}$ shown in Fig. 4; we indeed recover the poleward migration of the toroidal field, with a poleward branch at high latitudes being a little more pronounced than in case $\mathrm{SA}^{\prime}$. This was also one of the main differences between case $\mathrm{A}$ and case $\mathrm{B}$.

Figure 10 shows the comparison between the values of the toroidal and radial fields at a specific latitude and radius. The agreement is again very reasonable between all codes. Indeed, the difference between the various curves is hardly distinguishable if we do not zoom in on particular areas of the graphs. Zooming on the maximum of $B_{\phi}$ indicates that the deviations from the mean curve do not exceed $1.5 \%$ of the maximum value of the field. The phase shift is also very small, all curves recover the instant of zero-crossing quite well. If we do not take into account the extreme curve, which is a little further from the mean curve, we find the deviation does not exceed $0.6 \%$ of the mean value. This extremely small value indicates that the codes can reproduce very precisely the cycle period.

The agreement between all codes for the radial field at latitude $=30^{\circ}$ at the surface is reduced compared to the toroidal field at latitude $=60^{\circ}$ at the base of the convection zone. Nevertheless, the deviation from the mean curve stays under $3 \%$, despite the very sharp profile of $B_{\mathrm{r}}$ when it reaches its maximum. The deviations at the instant where $B_{\mathrm{r}}$ vanishes after one cycle do not exceed $3.5 \%$ of the cycle period. Most of the curves are located so close to each other that it is difficult to distinguish them even
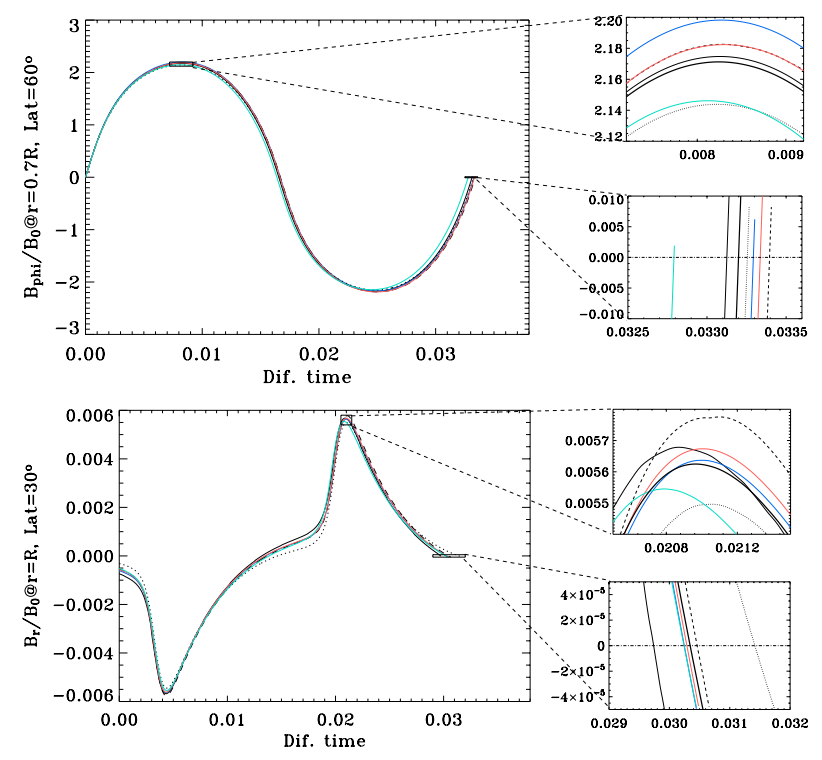

Fig. 10. Same as Fig. 5 but for case $\mathrm{SB}^{\prime}$.

on the zoomed frame, where the range represents only about $9 \%$ of the cycle period.

The agreement in cases $\mathrm{A}$ and $\mathrm{B}$ of $\alpha \Omega$ dynamos are thus very satisfactory, despite the strong gradients of $\alpha, \Omega$ and of magnetic diffusivity in case B. We now compare a completely different solar dynamo model which includes the meridional circulation, the large scale flow which is observed in the Sun and which may play a role in the dynamo loop.

\subsection{Case $C$}

Case $\mathrm{C}$ is a Babcock-Leighton flux-transport dynamo which incorporates a solar-like differential rotation, magnetic diffusivity sharply varying with depth, meridional circulation and a nonlocal Babcock-Leighton source term for the poloidal field. This model thus takes into account two observed solar features which may play a role in dynamo action: meridional flow and the twist of active regions at the surface. As we wish to be in the advection-dominated regime, we want an efficient connection between the magnetic field source regions (the base of the $\mathrm{CZ}$ and the surface) by means other than magnetic diffusion, i.e. the meridional circulation (see Eq. (19)). Moreover, in this model, the poloidal field owes its origin to the twisted nature of active regions at the solar surface due to the Coriolis force, which is again an observed feature of the solar magnetic activity. This mechanism is modeled by the non-local surface source term $S$ of Eq. (17) which appears in the equation for the evolution of $A_{\phi}$.

\subsubsection{Critical cases}

Looking at Table 4 , we notice that cases $\mathrm{C}$ and $\mathrm{C}^{\prime}$ require more spatial and temporal resolution for convergence than the $\alpha \Omega$ cases. A high resolution is needed to obtain a reasonably good agreement between all codes.

The imposition of radial field boundary conditions in case $\mathrm{C}^{\prime}$ can partly explain the high resolution needed. At the surface, the poloidal field and consequently $B_{\theta}$ is created and the outer boundary conditions force the component $B_{\theta}$ to be zero. The latitudinal component of $\boldsymbol{B}$ thus has to move smoothly from a non-zero value in a thin layer below the surface to zero at the 
Table 4. Same as for Table 2, for cases $\mathrm{C}$ and $\mathrm{C}^{\prime}$.

\begin{tabular}{cccccc}
\hline \hline Case & Code & Resolution & $\Delta t$ & $C_{\alpha}^{\text {crit }}$ & $\omega$ \\
\hline C & STELEM & $129 \times 129$ & $10^{-6}$ & 2.520 & 542 \\
C & NDYND & $81 \times 81$ & $10^{-6}$ & 2.513 & 525 \\
C & HAO2 & $101 \times 101$ & $10^{-5}$ & 2.515 & 546 \\
C & MBRK & $151 \times 151$ & $10^{-6}$ & 2.540 & 532 \\
C & HOLLER & $100 \times 100 *$ & $5 \times 10^{-8}$ & 2.355 & 538 \\
Mean val & & & & $\mathbf{2 . 4 8 9}$ & $\mathbf{5 3 6 . 6}$ \\
Std Dev. & & & & $\mathbf{0 . 0 7 5}$ & $\mathbf{8 . 2 9 5}$ \\
R. S. D. & & & & $\mathbf{0 . 0 3}$ & $\mathbf{0 . 0 1 5}$ \\
\hline$C^{\prime}$ & STELEM & $129 \times 129$ & $10^{-6}$ & 2.460 & 544 \\
CC$^{\prime}$ & NDYND & $321 \times 321$ & $5 \times 10^{-7}$ & 2.469 & 534 \\
CC$^{\prime}$ & MBRK & $256 \times 256$ & $5 \times 10^{-7}$ & 2.473 & 537 \\
CC$^{\prime}$ & MEFISTO & $201 \times 201$ & $10^{-6}$ & 2.463 & 539 \\
CC$^{\prime}$ & HAO1 & $128 \times 128$ & $3 \times 10^{-6}$ & 2.450 & 537 \\
Mean val & & & & $\mathbf{2 . 4 6 3}$ & $\mathbf{5 3 8 . 2}$ \\
Std Dev. & & & & $\mathbf{0 . 0 0 9}$ & $\mathbf{3 . 7 0 1}$ \\
R. S. D. & & & & $\mathbf{0 . 0 0 4}$ & $\mathbf{0 . 0 0 7}$ \\
\hline
\end{tabular}
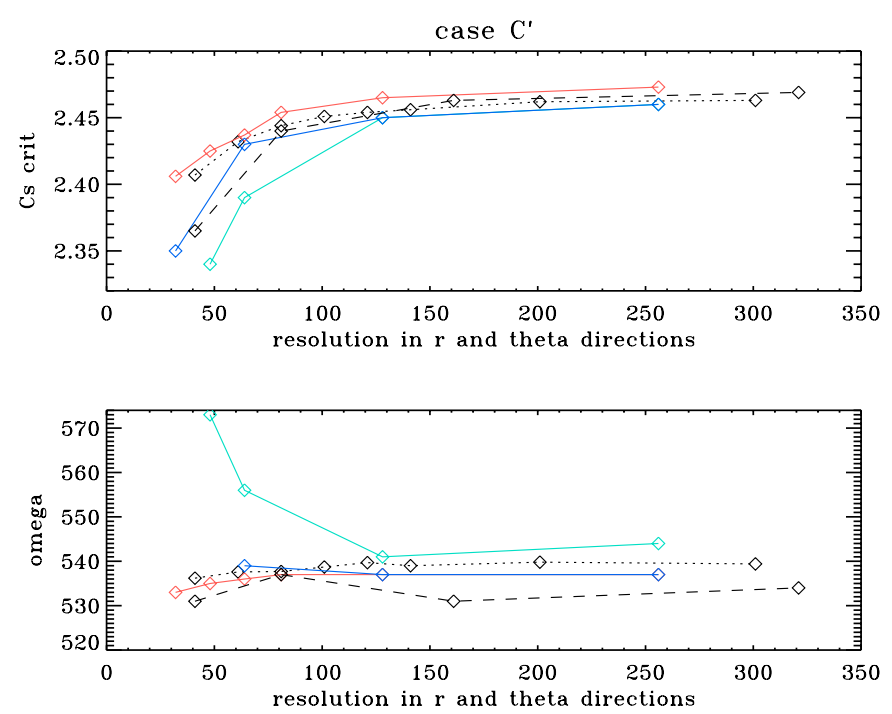

Fig. 11. Same as Fig. 6 but for case $C^{\prime}$. The colour coding is now the following: STELEM in green, NDYND in black dashed, MBRK in red, HAO1 in blue and MEFISTO in black dotted.

surface, which requires the resolution to be sufficient to handle this strong gradient.

The dispersion of the values for $C_{\mathrm{s}}^{\mathrm{crit}}$ in case $\mathrm{C}$ is higher than in the previous cases but the relative standard deviation is decreased from $3 \%$ to $0.5 \%$ if we remove the value of 2.355 of the HOLLERBACH code, which is quite far from the other results. The cycle frequency is also sensitive to the numerical method used, although all codes agree to within less than $2 \%$. We note that the agreement is increased in case $C^{\prime}$, when radial boundary conditions are used.

This more sophisticated flux-transport model (in comparison to the previous $\alpha \Omega$ models) leads to a higher though still reasonable dispersion of the values. This higher dispersion is to be expected, since this case includes a meridional flow and a non-local source term for the poloidal field and is therefore numerically more challenging.

Figure 11 shows the convergence behaviour of each code toward the values quoted in Table 4 for case $C^{\prime}$. Again, the agreement among the various codes is very satisfactory, the spread of the values in the converged part of the diagrams is less than $1 \%$

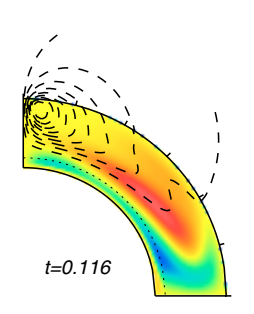

CASE C
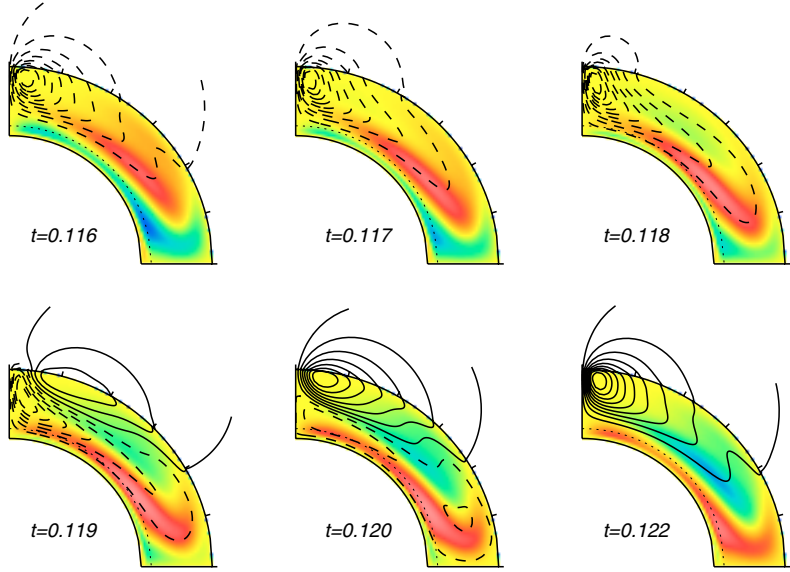

Fig. 12. Same as Fig. 2 but for case C: Babcock-Leighton flux-transport dynamo with a jump of magnetic diffusivity and with a meridional circulation of $10 \mathrm{~ms}^{-1}$ at the surface. The results are shown for $C_{\mathrm{s}}=C_{\mathrm{s}}^{\mathrm{crit}}$.

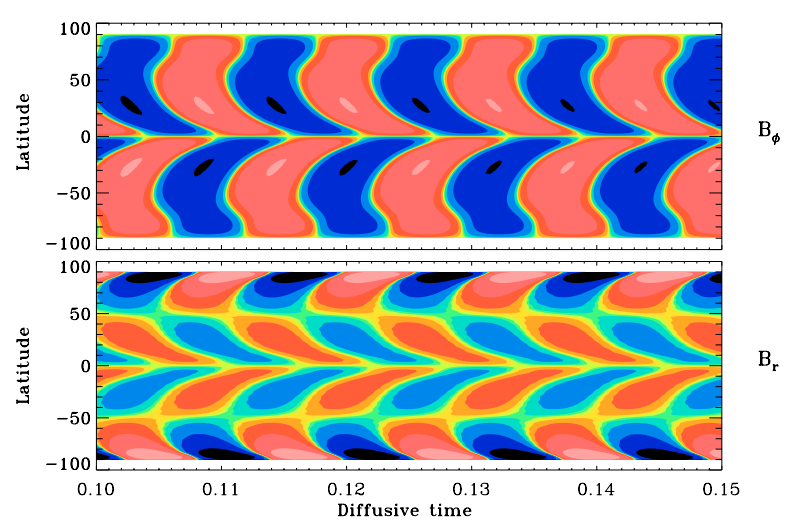

Fig. 13. Same as Fig. 3 but for case C.

of the absolute value. However, in this case, contrary to case B, most of the codes need a minimum resolution of $100 \times 100$ to be considered as converged. As stated before, since this case includes more sophisticated ingredients, it is numerically more challenging and more spatial resolution is required.

Figures 12 and 13 show the behaviour of the magnetic field in this Babcock-Leighton flux-transport model. We first notice that in this case we get an equatorward butterfly diagram, in agreement with the solar observations. Indeed, we find a very strong branch moving from $55^{\circ}$ latitude toward the equator, visible on the time-latitude cut of $B_{\phi}$ at the base of the CZ. Here the magnetic field follows the advective path created by the meridional flow and consequently this large scale flow plays a key role in these dynamo solutions. Figure 13 shows that the butterfly diagram of this BL case differs significantly from that of the previous $\alpha \Omega$ cases. Unlike cases $\mathrm{A}$ and $\mathrm{B}$, the field is not organized in very large and smooth structures; on the contrary the poloidal field is very concentrated near the poles in a narrow structure that will expand into lower latitudes as poloidal flux is advected poleward by the meridional circulation. The toroidal field, advected by the meridional flow, is also stretched in narrow bands, especially when it reaches the base of the CZ. The creation of these fine structures, organized in narrow latitudinal bands, causes strong field gradients to be created, which require both spatial and temporal resolution to be high. 


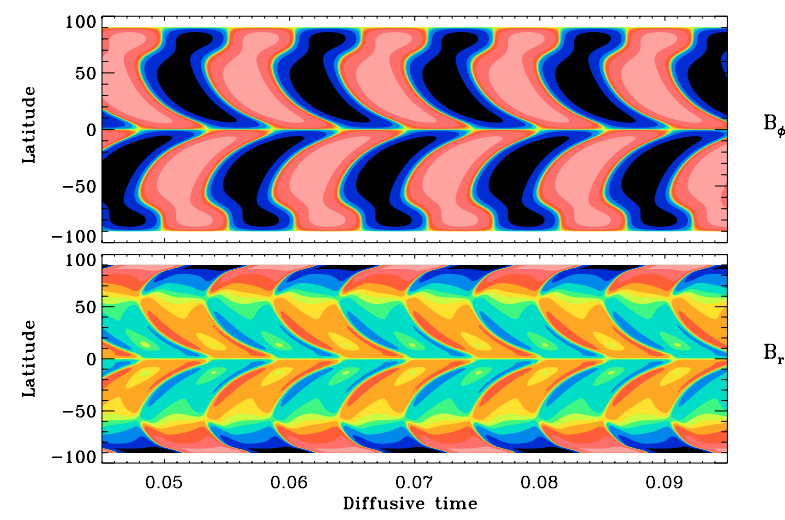

Fig. 14. Same as Fig. 4 but for case $S^{\prime}$.
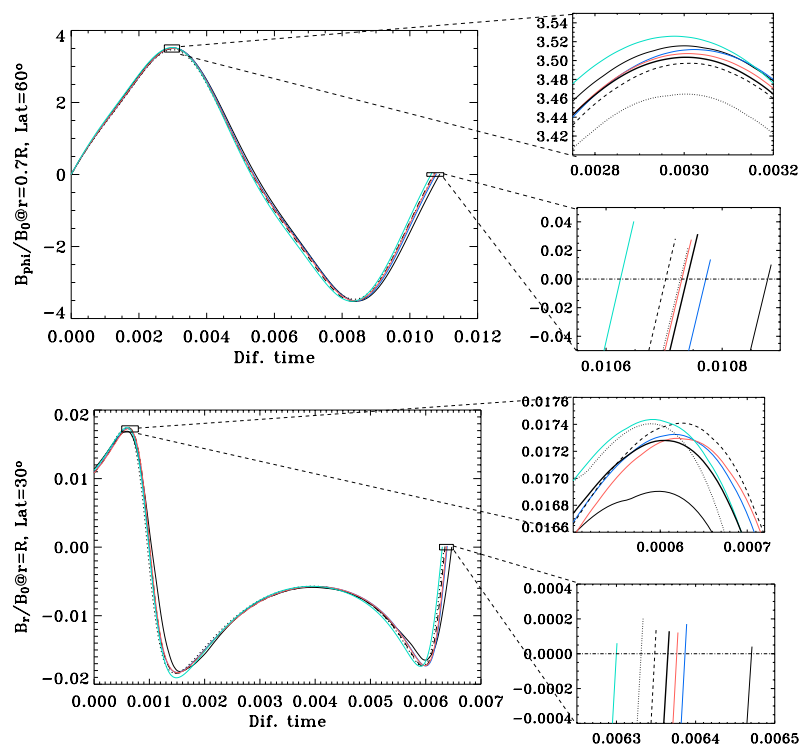

Fig. 15. Same as Fig. 5 but for case $\mathrm{SC}^{\prime}$.

\subsubsection{Supercritical case}

Increasing $C_{\mathrm{s}}$ to 35 (about 14 times supercritical for dynamo action) enables us to see the fields in the regime of a wellestablished flux-transport dynamo.

Figure 14 shows that the associated butterfly diagram for the toroidal field is similar to case $\mathrm{C}$ shown in Fig. 13. Some differences are nevertheless visible in the evolution of the radial field at the surface, like the appearance of finer structures close to the equator.

In this flux-transport model, the physical ingredients are more sophisticated and thus the uncertainty on the critical $C_{\mathrm{s}}$ and on the cycle period seems to be more significant. Nevertheless, as Fig. 15 shows, the agreement on the values of the toroidal and radial field at specific points is again very good. The deviation from the mean curve is of the order of $1 \%$ for both the maxima in the toroidal fields and for the differences in phase.

This time, the agreement on the behaviour of the radial field in one cycle period is as good as the agreement in the toroidal field. Both the maximum of the field and the instant where $B_{\mathrm{r}}$ vanishes are very well reproduced and are very close to the mean "optimal" solution.

\section{Conclusions}

Understanding the activity and magnetic cycle of the Sun is crucial since its variability has a direct impact on our technological society by disturbing high frequency signals, impairing satellites and damaging power grids. For decades the scientific community has developed observational, theoretical and numerical tools in order to be able to understand the inner workings of the Sun, while at the same time aiming to be able to predict its activity. The current accepted scenario states that this surface magnetism is linked to an internal dynamo. Two dimensional mean field dynamo models have proved to be very useful tools to test and validate ideas and distinguish among scenarios of the solar dynamo such as $\alpha \Omega, \alpha^{2}$ or flux-transport and to progress in our effort to understand the Sun as a whole.

The inclusion of the meridional circulation in the dynamo theory of the solar cycle turns out to be essential in reproducing the most important features of the 11-yr cycle. On the other hand if the eddy diffusivity $\eta_{\mathrm{t}}$ is as low as $10^{11} \mathrm{~cm}^{2} \mathrm{~s}^{-1}$, the magnetic Reynolds number $R_{\mathrm{e}}=u_{0} R_{\odot} / \eta_{\mathrm{t}}$ reaches values of the order of $10^{3}$ for a flow of about $10 \mathrm{~ms}^{-1}$.

How robust then are the predictions of dynamo codes for purely kinematic models? In this paper eight different codes, based on finite differences, finite elements and spectral decomposition, have been considered in order to solve the 2D induction equation in spherical geometry in the presence of strong gradients of the turbulent diffusivity, a realistic differential rotation profile and high magnetic Reynolds numbers. Again, the cases discussed in this paper are not intended to exhaustively cover all the solar dynamo models that have been published in the literature so far, such as non-linear Malkus-Proctor models (see e.g. Brandenburg et al. 1991; Moss \& Brooke 2000; or Bushby 2006). However, we believe that the cases chosen are representative of typical mean field kinematic dynamo models.

By defining three standard solar dynamo cases, namely cases A, B and C that are made increasingly more sophisticated by introducing well defined physical ingredients, we hope to have been able to provide useful test models easily reproducible by anyone seeking to validate their code. Cases A, B and C have potential boundary conditions on top of the domain. Similar cases are computed using radial field boundary conditions (cases $\mathrm{A}^{\prime}, \mathrm{B}^{\prime}$ and $\mathrm{C}^{\prime}$ ) since the imposition of $\mathrm{BCs}$ are known to be a delicate issue and is consequently a phase of code development that also needs to be carefully validated. For each case we list in summary tables the ingredients used, the critical dynamo number for the onset of dynamo action, and the resulting cycle period for each code. Moreover, we display typical realizations of the poloidal and toroidal fields in the meridional plane over half a cycle and butterfly diagrams of the toroidal field at the base of the convection zone as well as the radial field at the surface. In order to show that the solutions of these test cases are confirmed by several independent codes and are converged to high accuracy, we show convergence plots for two particular cases $B$ and $C^{\prime}$. Further, for each set of parameters chosen, we compute supercritical cases $\left(\mathrm{SA}^{\prime}, \mathrm{SB}^{\prime}\right.$ and $\mathrm{SC}^{\prime}$ ) including nonlinear quenching of the poloidal source term (the $\alpha$ effect for cases $\mathrm{A}$ and $\mathrm{B}$ and the Babcock-Leighton term for case C) at a fixed resolution of $101 \times 101$ and display the butterfly diagram obtained in that high dynamo number regime. In order to facilitate a more quantitative analysis of the results of the various codes, we plot for six of the eight codes used in this study specific cuts of the toroidal and radial fields at fixed latitude and radius. Given the quite good agreement between the different solutions obtained with the various numerical codes, these later 
figures serve two purposes: a) they help in determining the intrinsic deviation among all codes and b) they allow a quantitative comparison of the prediction made by the codes for the strength of the radial and toroidal fields in the numerical domain.

The key point to be extracted from our analysis is that the agreement between the different codes used in this benchmark study is indeed quite good, being of the order of (or even less than) $1 \%$ for the reference $\alpha \Omega$ dynamo cases $\mathrm{A}$ and $\mathrm{B}$, in spite of the non-trivial pattern of differential rotation and for case $B$ the presence of a strong gradient of the turbulent diffusivity. It is also important to note that in the search for critical values of the dynamo numbers, the eigenvalue code and the time-evolving codes agree rather well.

When the meridional flow is present as in case $\mathrm{C}$, the agreement is still reasonably good but with a dispersion of the order of a few percent in the values of the critical dynamo number and in the periods. This case includes meridional flow, a solar-like differential rotation, a sharp tachocline, a gradient of magnetic diffusivity and a non-local source term for the poloidal field. It is thus numerically more challenging and requires more resolution to converge, whatever the numerical technique used. Nevertheless the values of the toroidal field at the base of the convection zone and the butterfly diagrams are very well reproduced by all the codes for all the cases considered.

We anticipate that the well-documented benchmark cases presented here will be a useful tool for any researcher who intends to develop/validate his/her code, since they provide standard cases to which to refer and detailed tables and figures for a quantitative comparison.

Acknowledgements. We wish to thank the International Space Science Institute (ISSI) for supporting our international team with the program: observations and models of the solar cycle. M.D. acknowledges financial support from NASA grant NNH05AB521 and the NCAR Director's opportunity fund.

\section{References}

Ames, W. F. 1992, Numerical Methods for Partial Differential Equations, 3rd edn. (San Diego: Academic), Chap. 3

Arlt, R., Hollerbach, R., \& Rüdiger, G. 2003, A\&A, 401, 1087
Babcock, H. W. 1961, ApJ, 133, 572

Bonanno, A. 2004, Mem. Soc. Astron. It., 4, 17

Bonanno, A., Elstner, D., Rüdiger, G., \& Belvedere, G. 2002, A\&A, 390, 673

Brandenburg, A., Moss, D., Rüdiger, G., \& Tuominen, I. 1991, Geophys. Astrophys. Fluid Dyn., 61, 179

Brandenburg, A., Moss, D., \& Tuominen, I. 1992, A\&A, 265, 328

Browning, M. K., Miesch, M. S., Brun, A. S., \& Toomre, J. 2006, ApJ, 648, L157

Brun, A. S., Miesch, M. S., \& Toomre, J. 2004, ApJ, 614, 1073

Burnett, D. S., 1987, Finite Element Analysis (Addison-Wesley)

Bushby, P. J. 2006, MNRAS, 371, 772

Cattaneo, F. 1999, ApJ, 515, L39

Charbonneau, P. 2005, Liv. Rev. Sol. Phys., 2, 2

Choudhuri, A. R., Schüssler, M., \& Dikpati, M. 1995, A\&A, 303, L29

Dikpati, M. 1996, Ph.D. Thesis, Indian Ins. Sci., Bangalore

Dikpati, M., \& Charbonneau, P. 1999, ApJ, 518, 508

Dikpati, M., \& Gilman, P. A. 2006, ApJ, 649, 498

Dikpati, M., de Toma, G., Gilman, P. A., Arge, C. N., \& White, O. R. 2004, ApJ, 601,1136

Durney, B. R. 1995, Sol. Phys., 160, 213

Gilman, P. A. 1983, ApJS, 53, 243

Glatzmaier, G. A. 1987, The Internal Solar Angular Velocity, ed. B. R. Durney, \& S. Sofia (Dordrecht: D. Reidel), 263

Hollerbach, R. 2000, Int. J. Numer. Meth. Fluids, 32, 773

Jepps, S. A. 1975, J. Fluid Mech., 67, 625

Jouve, L., \& Brun, A. S. 2007, A\&A, 474, 239

Käpylä, P. J., Korpi, M. J., \& Tuominen, I. 2006, AN, 327, 884

Krause, F., \& Rädler, K. H. 1980, Mean Field Magnetohydrodynamics and Dynamo Theory (Oxford: Pergamon Press)

Küker, M., Rüdiger, G., \& Schultz, M. 2001, A\&A, 374, 301

Leighton, R. B. 1969, ApJ, 156, 1

Moffatt, H. K. 1978, Magnetic field Generation in Electrically Conducting Fluids (Cambridge University Press)

Moss, D., \& Brooke, J. 2000, MNRAS, 315, 521

Press, W. H., Teukolsky, S. A., Vetterling, W. T., \& Flannery, B. P. 1992, Numerical Recipes, 2nd edn. (Cambridge: Cambridge Univ.)

Proctor, M. R. E. 1977, J. Fluid Mech., 80, 769

Rempel, M. 2006, ApJ, 647, 662

Roberts, P. H. 1972, Phil. Trans. R. Soc. London, 272, 663

Spalart, P. R., Moser, R. D., \& Rogers, M. M. 1991, Comp. Phys., 96, 297

Steenbeck, M., \& Krause, F. 1969, AN, 291, 49

Steenbeck, M., Krause, F., \& Rädler, K. H. 1966, Z. Naturforsch. Teil A, 21, 369

Stix, M. 1976, A\&A, 47, 243

Wang, Y.-M., Sheeley, N. R., Jr., \& Nash, A. G. 1991, ApJ, 383, 431 